\title{
Evaluation of Response to Steroid Therapy for Cardiac Sarcoidosis Using Volumetric Analysis of ${ }^{18}$ F-FDG PET/CT
}

Yasuhiro Maruoka ${ }^{1 *}$, Michinobu Nagao ${ }^{2}$, Shingo Baba ${ }^{1}$, Takuro Isoda ${ }^{1}$, Yoshiyuki Kitamura ${ }^{1}$, Satoshi Kawanami ${ }^{1}$, Yuzo Yamasaki ${ }^{1}$, Masayuki Sasaki ${ }^{3}$, Tomomi Ide ${ }^{4}$, Kenichi Hiasa ${ }^{4}$ and Hiroshi Honda ${ }^{1}$

${ }^{1}$ Departments of Clinical Radiology, Graduate School of Medical Sciences, Kyushu University, Fukuoka, Japan

${ }^{2}$ Department of Diagnostic Imaging and Nuclear Medicine, Tokyo Women's Medical University

${ }^{3}$ Departments of Health Sciences, Graduate School of Medical Sciences, Kyushu University, Fukuoka, Japan

${ }^{4}$ Departments of Cardiovascular Medicine, Graduate School of Medical Sciences, Kyushu University, Fukuoka, Japan

\begin{abstract}
Background: The purpose of this study was to investigate the utility of total lesion glycolysis (TLG) in ${ }^{18} \mathrm{~F}$-fluorodeoxyglucose positron emission tomography/computed tomography $\left({ }^{18} \mathrm{~F}-\mathrm{FDG} \mathrm{PET} / \mathrm{CT}\right)$ to predict the response to steroid therapy for cardiac sarcoidosis (CS).

Methods: Thirty-six patients with clinically suspected CS who had undergone ${ }^{18} \mathrm{~F}$-FDG PET/CT were retrospectively analysed. Of the 36 patients, 21 were diagnosed as having CS according to Japanese Ministry of Health and Welfare guidelines and divided into 12 responders and 9 non-responders after steroid therapy by the mean follow-up period of 19 months. SUVmax and total lesion glycolysis (TLG) for the left ventricle (LV) on ${ }^{18}$ F-FDG $\mathrm{PET} / \mathrm{CT}$ were compared between responders and non-responders using the Wilcoxon test. The predictability of response to steroid therapy was analysed using receiver operating characteristic curve analysis.

Results: TLG for the LV wall was significantly higher in non-responders [1082 $\pm 715 \mathrm{~g}$ (mean $\pm \mathrm{SD}$ )] than in responders $(452 \pm 385 \mathrm{~g}, p=0.02)$, while there was no difference in the SUVmax for the LV wall between the two groups (responders $8.6 \pm 2.3$ vs. non-responders $11.4 \pm 3.8$ ). Use of an optimal TLG cut-off of $1070 \mathrm{~g}$ differentiated responders from non-responders with a sensitivity of $100 \%$, a specificity of $55.6 \%$, an accuracy of $81.0 \%$ and area under the curve of 0.80
\end{abstract}

Conclusion: The non-responders to steroid therapy for CS showed a high level of TLG on ${ }^{18} \mathrm{~F}-\mathrm{FDG}$ PET/CT. TLG of ${ }^{18} \mathrm{~F}-\mathrm{FDG}$ PET/CT can be a predictor of response to steroid therapy in CS.

Keywords: Cardiac sarcoidosis; Steroid therapy; Prediction; ${ }^{18} \mathrm{~F}-\mathrm{FDG}$ PET; Total lesion glycolysis

Abbreviations: CS: Cardiac Sarcoidosis; ${ }^{18} \mathrm{~F}-\mathrm{FDG}$ PET: ${ }^{18}$ F-Fluorodeoxyglucose Positron Emission Tomography; SUV: Standardized Uptake Value; MV: Metabolic Volume; TLG: Total Lesion Glycolysis; AVblock: Atrioventricular Block;ECG: Electrocardiogram; LV: Left Ventricle; JMHW guideline: Japanese Ministry of Health and Welfare Guideline; CRT: Cardiac Resynchronization Therapy; LVAD: Left Ventricle Assist Device; SUVmax: Maximum Standardized Uptake Value, VOI: Volume of Interest; RBBB: Right Bundle Branch Block; VT: Ventricular Tachycardia; PVC: Premature Ventricular Contraction; LVEF: Left Ventricle Ejection Fraction; SD: Standard Deviation; ROC curve analysis: Receiver Operating Characteristic Curve Analysis

\section{Introduction}

Sarcoidosis is a multisystem disease of unknown aetiology characterised by the presence of noncaseating granulomas that can affect different kinds of organs. Cardiac involvement in sarcoidosis is associated with heart failure, ventricular tachyarrhythmia, conduction disturbances, or sudden cardiac death and it is one of the major causes of disease-related death [1-4]. Steroid therapy is the mainstay of treating cardiac sarcoidosis (CS) [5,6] and its efficacy in general is about $50 \%[7,8]$. Corticosteroid-refractory CS patients require a change of therapeutic strategy, such as additional administration of immunosuppressants or placement of implantable cardiac defibrillators and have a poor prognosis [6]

${ }^{18} \mathrm{~F}$-fluorodeoxyglucose positron emission tomography $\left({ }^{18} \mathrm{~F}-\mathrm{FDG}\right.$ PET) is valuable for the diagnosis and detection of active inflammatory lesions in CS [9-11]. The utility of ${ }^{18} \mathrm{~F}-\mathrm{FDG}$ PET for the prediction of the therapeutic effect for CS or for the risk assessment of adverse events including sudden death has also been investigated $[9,12,13]$. On ${ }^{18} \mathrm{~F}-\mathrm{FDG}$ PET, the standardized uptake value (SUV) has been widely used as a quantitative index of the degree of ${ }^{18} \mathrm{~F}-\mathrm{FDG}$ uptake. In some studies of malignant disease, metabolic volume (MV) and total lesion glycolysis (TLG) were measured using the SUV of ${ }^{18} \mathrm{~F}-\mathrm{FDG}$ PET/ CT [14] and their prognostic significance has been reported [15-19]. Recent papers have reported metabolism-perfusion imaging to predict disease activity in CS with rubidium-FDG PET [20] or the usefulness of quantitative interpretation of ${ }^{18} \mathrm{~F}$-FDG PET in CS patients [21] However, the association between the responses to steroid therapy for CS patients and MV or TLG in ${ }^{18} \mathrm{~F}$-FDG PET remains unclear. The purpose of this study was to investigate the utility of TLG in ${ }^{18} \mathrm{~F}-\mathrm{FDG}$ PET/CT to predict the response to steroid therapy of CS [22].

\section{Materials and Methods}

\section{Patients}

Thirty-six consecutive patients with clinically suspected CS who

*Corresponding author: Yasuhiro Maruoka, Department of Clinical Radiology Graduate School of Medical Sciences, Kyushu University, Fukuoka, Japan, Tel: (81)92-642-5695; Fax: (81)-92-642-5706; E-mail: ymaruoka@radiol.med.kyushu-u.ac.jp

Received September 11, 2017; Accepted September 22, 2017; Published September 29, 2017

Citation: Maruoka Y, Nagao M, Baba S, Isoda T, Kitamura Y, et al. (2017) Evaluation of Response to Steroid Therapy for Cardiac Sarcoidosis Using Volumetric Analysis of ${ }^{18} \mathrm{~F}-\mathrm{FDG}$ PET/CT. J Bioanal Biomed 9: 229-234. doi:10.4172/1948-593X.1000184

Copyright: @ 2017 Maruoka Y, et al. This is an open-access article distributed under the terms of the Creative Commons Attribution License, which permits unrestricted use, distribution, and reproduction in any medium, provided the original author and source are credited. 
had undergone ${ }^{18} \mathrm{~F}$-FDG PET/CT for diagnosis between January 2010 and December 2013 were retrospectively analysed. The entry criteria were as follows: (1) atrioventricular (AV) block on electrocardiogram (ECG) or left ventricular (LV) wall motion abnormality and septal wall thinning on echocardiography; or (2) histologically proven sarcoidosis in lung, muscle and other organs. The exclusion criteria were as follows: (1) known coronary artery disease, myocarditis, valvular heart disease, or cardiomyopathies other than CS; and (2) high blood glucose level $(>150 \mathrm{mg} / \mathrm{dL})$. This retrospective study was approved by the institutional review board and the requirement for written informed consent was waived.

A total of 36 patients were classified into two groups of CS patients and non-CS patients based on 2006 Japanese Ministry of Health and Welfare (JMHW) guidelines [22,23]. The patients' characteristics are shown in Table 1. In all patients, an ${ }^{18} \mathrm{~F}$-FDG PET/CT examination was performed to make the diagnosis before treatment. In CS patients, the treatment started with prednisolone $30 \mathrm{mg}$ per day. Twelve months after initiation of steroid therapy, the response was determined by the consensus of two cardiologists. CS patients in whom the corticosteroid dose could be tapered due to stable clinical symptoms or improved cardiac function were defined as responders. CS patients who required additional immunosuppressant or placement of cardiac resynchronization therapy (CRT) or a LV assist device (LVAD) because of unstable symptoms or progression of cardiac dysfunction were defined as non-responders. All responders were maintained on $5 \mathrm{mg}$ to $10 \mathrm{mg}$ of prednisolone per day and had no recurrence of CS during follow-up, which ranged from 17 months to 45 months (mean, 25 months). ${ }^{18} \mathrm{~F}-\mathrm{FDG}$ PET/CT was again performed for 17 of $21 \mathrm{CS}$ patients to evaluate active lesions after initiation of steroid therapy (mean, 8 months).

\section{${ }^{18}$ F-FDG PET/CT Imaging}

In each patient, $185 \mathrm{MBq}$ of ${ }^{18} \mathrm{~F}-\mathrm{FDG}$ was intravenously administered after 18 -hr fasting. ${ }^{18} \mathrm{~F}$-FDG PET/CT with an intravenous injection of heparin $(50 \mathrm{IU} / \mathrm{kg}) 15 \mathrm{~min}$ before ${ }^{18} \mathrm{~F}$-FDG injection was performed. Scans of the heart were conducted $60 \mathrm{~min}$ after ${ }^{18} \mathrm{~F}-\mathrm{FDG}$ administration. ${ }^{18} \mathrm{~F}-\mathrm{FDG}$ PET/CT images were obtained using an integrated PET/CT scanner Discovery STE (GE Medical Systems, Milwaukee, WI). The PET scanner comprised 24 ring detectors consisting of 560 BGO crystals $(4.7 \times 6.3 \times 30 \mathrm{~mm})$. All emission scans were performed in 3 -dimensional mode with $128 \times 128$ matrices $(5.47 \times 5.47 \times 3.27 \mathrm{~mm})$ and the acquisition time per bed position was $10 \mathrm{~min}$. The PET images were reconstructed using the orderedsubset expectation-maximization method (VUE Point Plus) with 2 full iterations of 28 subsets and full-width at half maximum was $5.2 \mathrm{~mm}$. A low-dose 16-slice CT (tube voltage, $120 \mathrm{kV}$; effective tube current, $30-250 \mathrm{~mA}$ ) that covered from the vertex to the proximal thigh was performed for attenuation correction and for determining the precise anatomic location before acquisition of the PET image. The CT scan was reconstructed by filtered back projection into $512 \times 512$ pixel images with a slice thickness of $5 \mathrm{~mm}$ to match the PET scan.

\section{TLG of ${ }^{18}$ F-FDG PET/CT}

Maximum SUV (SUVmax), MV and TLG in ${ }^{18} \mathrm{~F}-\mathrm{FDG}$ PET imaging were measured by the available software (Multi-Modality Tumor Tracking) in a dedicated workstation (IntelliSpace Portal 6, Philips Medical Systems, Milpitas, CA). SUV was obtained from each pixel as pixel activity (injected dose/body weight). A spherical volume of interest (VOI) corresponding to the entire LV wall was manually drawn and ${ }^{18} \mathrm{~F}-\mathrm{FDG}$ uptake except for the LV wall was excluded. SUVmax for the VOI was automatically calculated. Then, an SUV of 4.0 for the LV wall was determined as a low cut-off threshold. The volume for the LV wall with SUV $\geq 4.0$ was then measured as MV. TLG was calculated by dividing the MV by the mean SUV in the MV.

\section{ECG data analysis}

Resting 12-lead ECG or $24 \mathrm{~h}$ ECG monitoring was analysed by cardiologists blinded to ${ }^{18} \mathrm{~F}$-FDG PET data. According to the JMHW guidelines, right bundle branch block (RBBB), AV block, left-axis deviation, ventricular tachycardia (VT), premature ventricular contraction (PVC) (grade 2 in Lown's classification) and abnormal Q or ST-T changes were defined as abnormal.

\section{Echocardiography data analysis}

All patients underwent transthoracic or transesophageal echocardiography and their data were analysed by cardiologists blinded to ${ }^{18} \mathrm{~F}$-FDG PET data. According to the JMHW guidelines, abnormal wall motion, regional wall thinning or thickening and dilatation of the left ventricle were defined as abnormal. The LV ejection fraction (LVEF) was also measured by analysis of the LV end-diastolic and endsystolic dimensions using M-mode or B-mode echocardiography.

\section{Major adverse cardiac events during follow-up}

Patients were examined by cardiologists in our hospital at least every 3 months for a mean follow-up period of 25 months (17 months to 45 months). The primary endpoint was major adverse cardiac events consisting of cardiac death or heart failure hospitalization for CRT or LVAD.

\section{Statistical analysis}

Continuous data are expressed as means \pm standard deviation (SD). Comparisons of LVEF, SUVmax, MV and TLG between responders and non-responders were analysed by the Wilcoxon test. The ability of SUVmax, MV and TLG to differentiate CS patients from non-CS patients and to predict the response to therapy was analysed by receiver operating characteristic (ROC) curve analysis. Comparisons of parameters between pre- and post-steroid therapy were performed using paired $t$-tests. Survival curves of patient subgroups were created by the Kaplan-Meier method to clarify the time-dependent, cumulative event-free rate and were compared using the log-rank test. The tests were performed using JMP' (version 9.0.2; SAS Institute, Cary, NC) statistical software. A $p$ value of less than 0.05 was considered significant.

\section{Results}

\section{Comparison of ${ }^{18}$ F-FDG PET measurements between CS and non-CS patients}

On the basis of the JMHW guidelines, 21 of the 36 patients were diagnosed as having CS and the other 15 patients were diagnosed as not having CS (non-CS patients). CS and non-CS patients' characteristics are shown in Table 1. The SUVmax, MV and TLG were significantly greater for CS patients than for non-CS patients ( $p<0.01$; Table 2$)$.

\section{Diagnostic capability of CS patients by ${ }^{18} \mathrm{~F}-\mathrm{FDG}$ PET measurements}

ROC curve analysis revealed that the optimal SUVmax was 5.3 for differentiating CS patients from non-CS patients, with an AUC of 1.0, $100 \%$ accuracy $(36 / 36), 100 \%$ sensitivity $(21 / 21)$ and $100 \%$ specificity (15/15). The optimal MV and TLG thresholds were $4 \mathrm{~cm}^{3}$ and $16 \mathrm{~g}$ for differentiating CS patients from non-CS patients, with an AUC of 0.99 


\begin{tabular}{|l|c|c|}
\hline & $\begin{array}{c}\text { CS patients } \\
\text { (n=21) }\end{array}$ & $\begin{array}{c}\text { Non-CS patients } \\
\text { (n=15) }\end{array}$ \\
\hline Age (years) & $61 \pm 11$ & $59 \pm 14$ \\
\hline Sex (male/female) & $7 / 14$ & $5 / 10$ \\
\hline Diabetes & $1(\%)$ & $0(0 \%)$ \\
\hline Electrocardiographic abnormalities & -- & -- \\
\hline Atrioventricular block & $13(62 \%)$ & $1(7 \%)$ \\
\hline Left bundle branch block & $2(10 \%)$ & $3(20 \%)$ \\
\hline Right bundle branch block & $10(48 \%)$ & $2(13 \%)$ \\
\hline Left axis deviation & $3(14 \%)$ & $0(0 \%)$ \\
\hline Premature ventricular contraction & $10(48 \%)$ & $5(33 \%)$ \\
\hline Ventricular tachycardia & $2(10 \%)$ & $1(7 \%)$ \\
\hline Abnormal Q wave & $5(24 \%)$ & $0(0 \%)$ \\
\hline ST-T abnormalities & $3(14 \%)$ & $2(13 \%)$ \\
\hline Echocardiography abnormalities & -- & -- \\
\hline Interventricular septum wall thinning & $11(52 \%)$ & $5(33 \%)$ \\
\hline Regional wall motion abnormality & $21(100 \%)$ & $10(67 \%)$ \\
\hline Ventricular aneurysm & $3(14 \%)$ & $17(\%)$ \\
\hline Regional wall thickening & $4(19 \%)$ & $0(0 \%)$ \\
\hline Left ventricular ejection fraction $(\%)$ & $40 \pm 12{ }^{*}$ & $57 \pm 10$ \\
\hline Extra cardiac sarcoidosis & $19(90 \%)$ & $5(33 \%)$ \\
\hline Lymph node & $19(90 \%)$ & $5(33 \%)$ \\
\hline Lung & $9(43 \%)$ & $5(33 \%)$ \\
\hline Eye & $8(38 \%)$ & $3(30 \%)$ \\
\hline Liver & $4(19 \%)$ & $1(7 \%)$ \\
\hline Spleen & $4(19 \%)$ & $1(7 \%)$ \\
\hline Skin & $2(10 \%)$ & $0(0 \%)$ \\
\hline Bone & $0(0 \%)$ & $1(7 \%)$ \\
\hline Data are mean \pm SD or number of patients; & & \\
\hline CS: Cardiac Sarcoidosis; & & \\
\hline P<0.01 vs. non-CS patients. & & \\
\hline
\end{tabular}

Table 1: Patient characteristics.

\begin{tabular}{|c|c|c|}
\hline & CS patients $(n=21)$ & $\begin{array}{c}\text { Non-CS patients } \\
(n=15)\end{array}$ \\
\hline${ }^{18} \mathrm{~F}-\mathrm{FDG}$ accumulation of $\mathrm{LV}$ wall & -- & -- \\
\hline SUVmax & $9.8 \pm 3.3^{*}$ & $2.8 \pm 1.0$ \\
\hline Metabolic volume $\left(\mathrm{cm}^{3}\right)$ & $127 \pm 101^{*}$ & $8 \pm 2$ \\
\hline Total lesion glycolysis (g) & $722 \pm 623^{*}$ & $35 \pm 9$ \\
\hline Fasting blood glucose $(\mathrm{mg} / \mathrm{dL})$ & $97 \pm 15$ & $99 \pm 12$ \\
\hline
\end{tabular}

Table 2: Comparison of ${ }^{18} \mathrm{~F}-\mathrm{FDG} \mathrm{PET} / \mathrm{CT}$ measurements between $\mathrm{CS}$ and non-CS patients.

and $0.99,97 \%(35 / 36)$ and $97 \%(35 / 36)$ accuracy, $100 \%(21 / 21)$ and $100 \%(21 / 21)$ sensitivity and $93 \%(14 / 15)$ and $93 \%(14 / 15)$ specificity, respectively.

\section{Comparison of ${ }^{18}$ F-FDG PET measurements and LVEF between responders and non-responders}

Of the 21 CS patients, 12 patients were responders to steroid therapy and the other 9 were non-responders. MV and TLG were significantly greater for non-responders than for responders $\left(182 \pm 112 \mathrm{~cm}^{3} \mathrm{vs} .85\right.$ $\pm 69 \mathrm{~cm}^{3} ; p=0.03,1082 \pm 715 \mathrm{~g}$ vs. $\left.452 \pm 385 \mathrm{~g} ; p=0.02\right)$, respectively (Figure 1). No significant differences in LVEF (responder's $42 \% \pm 13 \%$ vs. non-responders $37 \% \pm 10 \%$ ) and SUVmax (responders $8.6 \pm 2.3$ vs. non-responders $11.4 \pm 3.8$ ) were noted between the responders and non-responders.

\section{Predictability of response to steroid therapy in CS patients}

ROC curve analysis revealed that the optimal SUVmax was 8.0 for predicting non-responders, with an AUC of 0.69, 66.7\% accuracy (14/21), $58.3 \%$ sensitivity (7/12) and $77.8 \%$ specificity (7/9). The optimal MV and TLG thresholds were $190 \mathrm{~cm}^{3}$ and $1070 \mathrm{~g}$ for predicting non-responders, with an AUC of 0.79 and $0.80,81 \%(17 / 21)$ and $81 \%(17 / 21)$ accuracy, $100 \%(21 / 21)$ and 100\% (21/21) sensitivity and 56\% (5/9) and 56\% (5/9) specificity (5/9), respectively.

\section{Comparison of ${ }^{18}$ F-FDG PET measurements between before and after therapy}

In CS responders, SUVmax $(8.4 \pm 1.8$ vs. $3.8 \pm 1.0)$, MV (69 $\pm 50 \mathrm{~cm}^{3}$ vs. $\left.2 \pm 3 \mathrm{~cm}^{3}\right)$ and TLG $(346 \pm 281 \mathrm{~g}$ vs. $10 \pm 16 \mathrm{~g})$ were significantly greater at pre-therapy than at post-therapy (Figure 2). In non-responders, there were no significant differences in SUVmax, MV and TLG between the two conditions.

Representative ${ }^{18} \mathrm{~F}-\mathrm{FDG}$ PET images at pre and post-steroid therapy for non-responders and responders are presented in Figures 3 and 4.

\section{${ }^{18}$ F-FDG PET measurements and major adverse cardiac events}

After initiation of steroid therapy, 5 of 21 CS patients had implantation of CRT (after $2 \pm 1$ month) and 1 patient had implantation of LVAD (after 1 month). Event-free rates had no significant difference between patients with $\mathrm{SUVmax}<8.0$ and $\geq 8.0$ (Log-rank value $=0.30$, $p=0.58$ ). On the other hand, $M V<190 \mathrm{~cm}^{3}$ and TLG $<1070 \mathrm{~g}$ had significantly lower event-free rates than those with $\geq 190 \mathrm{~cm}^{3}$ (Logrank value=9.93, $p<0.01)$ and $\geq 1070 \mathrm{~g}$ (Log-rank value=9.93, $p<0.01)$, respectively (Figure 5).

\section{Discussion}

Our results demonstrated that all of the SUVmax, MV and TLG in LV wall on ${ }^{18}$ F-FDG PET were significantly higher in CS patients than in non-CS patients and had high diagnostic capability for CS. In our study, $18 \mathrm{~h}$ fasting before ${ }^{18} \mathrm{~F}$-FDG injection was performed, which have been known as a useful method to inhibit physiological myocardial uptake [23]. On diagnostic performance of CS, it was suggested that the SUVmax in LV wall was no less effective than both MV and TLG in the condition of sufficient suppression of physiological myocardial uptake.

On the contrary, the MV and TLG had significantly higher predictive value for the response to steroid therapy and adverse cardiac events than the SUVmax. That suggests that the response to steroid therapy in CS patients is associated with the extent as well as the degree of active inflammation in the LV wall before therapy. We think that MV or TLG is associated with the extent of active inflammation in CS, as well as the extent or volume of malignant tumour. Therefore, MV or TLG is superior to SUVmax in predicting the response to steroid therapy. In addition, after steroid therapy, the responders showed almost no remaining MV and TLG, while non-responders had no significant decrease of either. The assessment of MV and TLG in the LV wall after treatment for CS can clarify the presence or absence of continuing active inflammation. That suggests that ${ }^{18} \mathrm{~F}-\mathrm{FDG}$ PET is a helpful tool for evaluation after, as well as before, steroid therapy for CS.

The most important factor is the determination of the lower cutoff of myocardial uptake in the process of calculating TLG. In the present results, the mean SUVmax for LV in non-CS patients was 2.8. A previous $18 \mathrm{~h}$ fasting ${ }^{18} \mathrm{~F}$-FDG PET study showed a SUVmax of 4.2 for active inflammatory myocardium in CS [24]. The present ${ }^{18} \mathrm{~F}-\mathrm{FDG}$ PET protocol involved $18 \mathrm{~h}$ fasting and heparin loading to suppress 
Citation: Maruoka Y, Nagao M, Baba S, Isoda T, Kitamura Y, et al. (2017) Evaluation of Response to Steroid Therapy for Cardiac Sarcoidosis Using Volumetric Analysis of ${ }^{18}$ F-FDG PET/CT. J Bioanal Biomed 9: 229-234. doi:10.4172/1948-593X.1000184
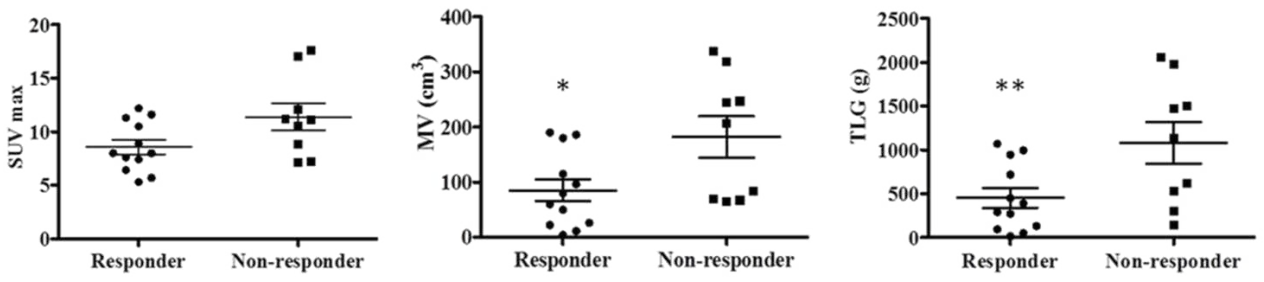

Figure 1: The box-and-whisker plot of ${ }^{18} \mathrm{~F}$-FDG PET measurements in responders and non-responders. The boxes represent the $25 \%$ to $75 \%$ range with bisecting lines showing the median value and the horizontal lines represent the $10 \%$ to $90 \%$ range. SUVmax (left) of the LV wall shows no significant difference between responders and non-responders. The MV (centre) of the LV wall is significantly higher in non-responders than in responders. ${ }^{*} P=0.03$. TLG (right) of the LV wall is significantly higher in non-responders than in responders. ${ }^{* *} P=0.02$.
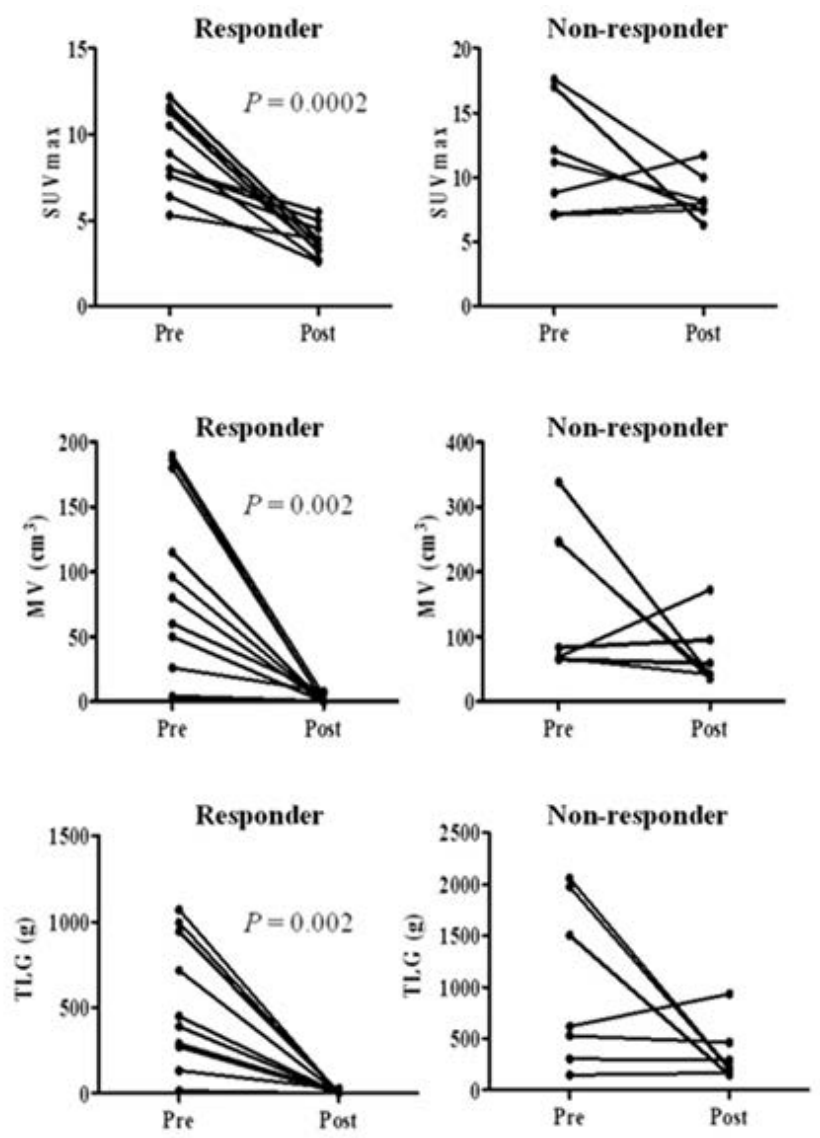

Figure 2: Comparison of ${ }^{18} \mathrm{~F}-\mathrm{FDG}$ PET measurements between before and after steroid therapy. The graphs of data points and fitted lines in ${ }^{18} \mathrm{~F}-\mathrm{FDG}$ PET measurements before and after steroid therapy are presented. In CS responders, SUVmax (upper), MV (middle) and TLG (lower) are significantly greater at pre-therapy than at post-therapy. In non-responders, there are no significant differences in SUVmax, MV and TLG between the two conditions.

physiological myocardial ${ }^{18} \mathrm{~F}-\mathrm{FDG}$ uptake. According to these data, the lower cut-off of SUV for normal myocardium was determined and the TLG was calculated to represent the active inflammatory area. Our proposed method is a feasible and quantitative method to extract the active inflammatory lesion and suppress physiological myocardial uptake in CS.

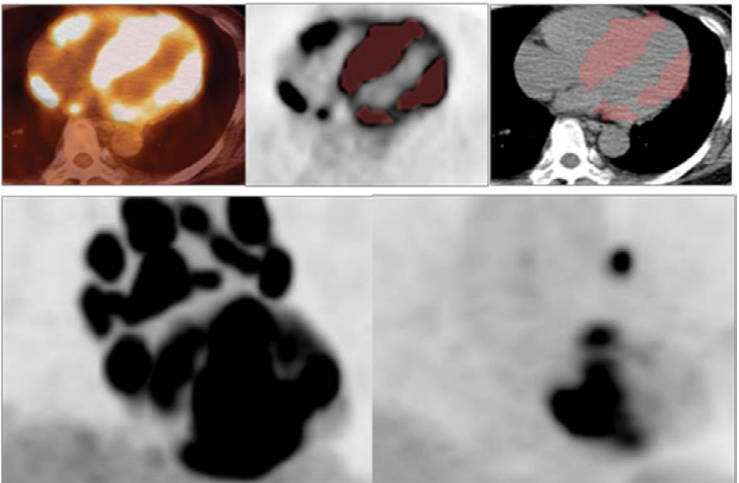

Figure 3: A 54-year-old female who was a non-responder to steroid therapy for CS. From ${ }^{18} \mathrm{~F}-\mathrm{FDG}$ PET/CT fusion images before steroid therapy (upper left) the volume of the LV wall with SUV $\geq 4.0$ automatically extracted (upper centre) and displayed on the CT images (upper right). The SUVmax, MV and TLG in the LV wall before therapy are $17.6,245 \mathrm{~cm}^{3}$ and $1976 \mathrm{~g}$, respectively. Maximumintensity-projection image of ${ }^{18} \mathrm{~F}-\mathrm{FDG}$ PET/CT before steroid therapy (lower left) and 8 months after initiation of steroid therapy (lower right) are presented. After steroid therapy, the patient had continually unstable symptoms of heart failure and cardiac dysfunction and additional administration of methotrexate was performed. The post-therapy SUVmax, MV and TLG in the LV wall after steroid therapy are $10.0,42 \mathrm{~cm}^{3}$ and $282 \mathrm{~g}$, respectively.

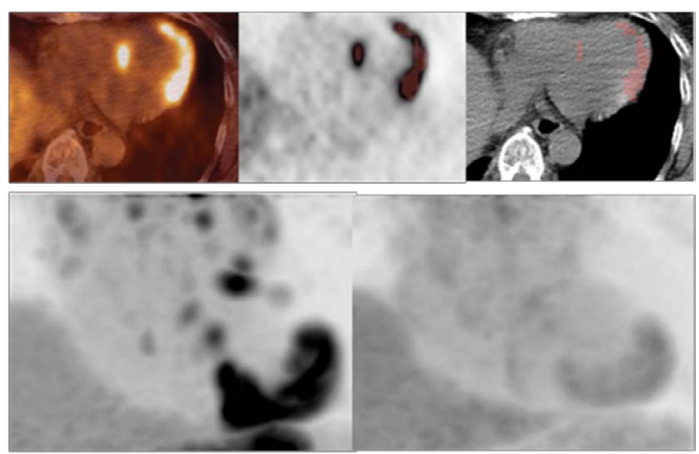

Figure 4: A 66-year-old male who was a responder to steroid therapy for CS. From ${ }^{18} \mathrm{~F}-\mathrm{FDG}$ PET/CT fusion images before steroid therapy (upper left), the volume of the LV wall with SUV $\geq 4.0$ is automatically extracted (upper centre) and displayed on the CT images (upper right). The SUVmax, MV and TLG in the LV wall are $11.3,50 \mathrm{~cm}^{3}$ and $391 \mathrm{~g}$, respectively. SUVmax is high, suggesting a non-responder. However, TLG could predict a responder. Maximum-intensityprojection images of ${ }^{18} \mathrm{~F}$-FDG PET/CT before steroid therapy (lower left) and 5 months after initiation of steroid therapy (lower right) are presented. The patient has improvement of heart failure and ${ }^{18} \mathrm{~F}-\mathrm{FDG}$ accumulation of the LV wall has almost disappeared. The SUVmax and TLG in the LV wall after steroid therapy are $3.2,0 \mathrm{~cm}^{3}$ and $0 \mathrm{~g}$, respectively. 
Citation: Maruoka Y, Nagao M, Baba S, Isoda T, Kitamura Y, et al. (2017) Evaluation of Response to Steroid Therapy for Cardiac Sarcoidosis Using Volumetric Analysis of ${ }^{18}$ F-FDG PET/CT. J Bioanal Biomed 9: 229-234. doi:10.4172/1948-593X.1000184
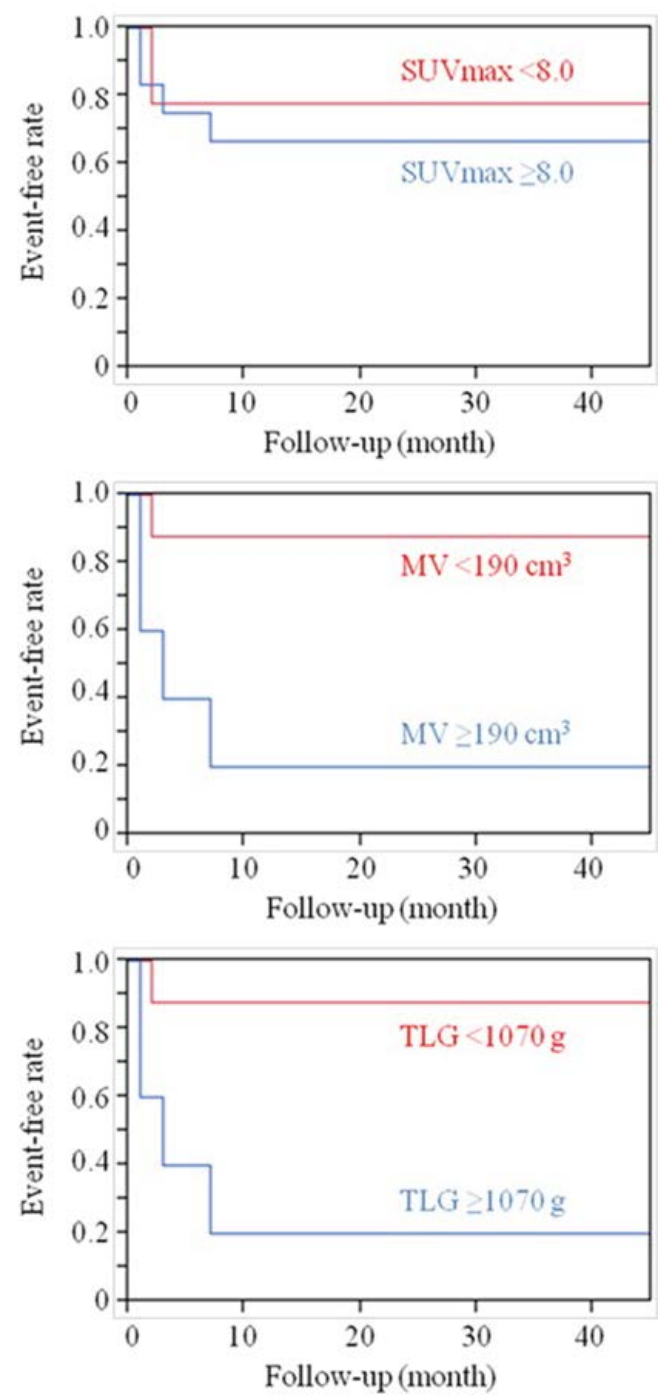

Figure 5: Event-free curves of two groups classified by cut-offs of SUVmax $<8.0$ and $\geq 8.0$ (upper), MV $<190 \mathrm{~cm}^{3}$ and $\geq 190 \mathrm{~cm}^{3}$ (middle) and TLG $<1070 \mathrm{~g}$ and $\geq 1070 \mathrm{~g}$ (lower). Event-free rates had no significant difference between patients with SUVmax $<8.0$ (in red) and $\geq 8.0$ (in blue). $M V<190 \mathrm{~cm}^{3}$ (in red) and TLG $<1070 \mathrm{~g}$ (in red) had significantly lower event-free rates than those with $\geq$ $190 \mathrm{~cm}^{3}$ (in blue) and $\geq 1070 \mathrm{~g}$ (in blue), respectively.

The present study had several limitations. First, the mean follow-up period was 25 months and the present study lacked long-term followup data to confirm the outcomes of responders to steroid therapy in CS. Second, the number of CS patients analysed in the present study was relatively small in a single centre. Further studies are needed to confirm our hypothesis by evaluating the outcomes of CS patients during longterm follow-up in multicentre studies.

\section{Conclusion}

In conclusion, TLG, which expresses metabolic and volumetric indices in ${ }^{18} \mathrm{~F}$-FDG PET, was significantly greater in non-responders than in responders to steroid therapy for CS. TLG is more useful than SUVmax in the prediction of the response to steroid therapy for CS and contributes to clinical management and determination of the therapeutic strategy in CS patients.

\section{References}

1. Iannuzzi MC, Rybicki BA, Teirstein AS (2007) Sarcoidosis. N Engl J Med 357: 2153-2165.

2. Rybicki BA, Major M, Popovich Jr J, Maliarik MJ, lannuzzi MC (1997) Racial differences in sarcoidosis incidence: A 5-year study in a health maintenance organization. Am J Epidemiol 145: 234-241.

3. Doughan AR, Williams BR (2006) Cardiac sarcoidosis. Heart 92: 282-288

4. Mehta D, Lubitz SA, Frankel Z, Wisnivesky JP, Einstein AJ, et al. (2008) Cardiac involvement in patients with sarcoidosis: Diagnostic and prognostic value of outpatient testing. Chest 133: 1426-1435

5. Dubrey SW, Falk RH (2010) Diagnosis and management of cardiac sarcoidosis Prog Cardiovasc Dis 52: 336-346.

6. Yazaki Y, Isobe M, Hiroe M, Morimoto S, Hiramitsu S, et al. (2001) Prognostic determinants of long-term survival in Japanese patients with cardiac sarcoidosis treated with prednisone. Am J Cardiol 88: 1006-1010.

7. Sugisaki K, Yamaguchi T, Nagai S, Ohmiti M, Takenaka S, et al. (2003) Clinical characteristics of 195 Japanese sarcoidosis patients treated with ora corticosteroids. Sarcoidosis Vasc Diffuse Lung Dis 20: 222-226.

8. Hiramitsu S, Morimoto S, Uemura A, Kato Y, Kimura K, et al. (2005) National survey on status of steroid therapy for cardiac sarcoidosis in Japan. Sarcoidosis Vasc Diffuse Lung Dis 22: 210-213.

9. Ishimaru S, Tsujino I, Takei T, Tsukamoto E, Sakaue S, et al. (2005) Foca uptake on 18F-fluoro-2-deoxyglucose positron emission tomography images indicates cardiac involvement of sarcoidosis. Eur Heart J 26: 1538-1543.

10. Ohira H, Tsujino I, Yoshinaga $\mathrm{K}(2011){ }^{18} \mathrm{~F}$-fluoro-2-deoxyglucose positron emission tomography in cardiac sarcoidosis. Eur J Nucl Med Mol Imaging 38 $1558-1566$.

11. Youssef G, Leung E, Mylonas I, Nery P, Williams K, et al. (2012) The use of 18F-FDG PET in the diagnosis of cardiac sarcoidosis: a systematic review and metaanalysis including the Ontario experience. J Nucl Med 53: 241-248.

12. Osborne MT, Hulten EA, Singh A, Waller AH, Bittencourt MS, et al. (2014) Reduction in ${ }^{18} \mathrm{~F}$-fluorodeoxyglucose uptake on serial cardiac positron emission tomography is associated with improved left ventricular ejection fraction in patients with cardiac sarcoidosis. J Nucl Cardiol 21: 166-174.

13. Blankstein R, Osborne M, Naya M, Waller A, Kim CK, et al. (2014) Cardiac positron emission tomography enhances prognostic assessments of patients with suspected cardiac sarcoidosis. J Am Coll Cardiol 63: 329-336.

14. Larson SM, Erdi Y, Akhurst T, Mazumdar M, Macapinlac HA, et al. (1999) Tumor treatment response based on visual and quantitative changes in global tumor glycolysis using PET-FDG imaging: The visual response score and the change in total lesion glycolysis. Clin Positron Imaging 2: 159-171.

15. Oh JR, Seo JH, Chong A, Min JJ, Song HC, et al. (2012) Whole-body metabolic tumour volume of $18 \mathrm{~F}-\mathrm{FDG}$ PET/CT improves the prediction of prognosis in small cell lung cancer. Eur J Nucl Med Mol Imaging 39: 925-935.

16. Yan H, Wang R, Zhao F, Zhu K, Jiang S, et al. (2011) Measurement of tumor volume by PET to evaluate prognosis in patients with advanced non-small cell lung cancer treated by nonsurgical therapy. Acta Radiol 52: 646-650.

17. Byun BH, Kong CB, Park J, Seo Y, Lim I, et al. (2013) Initial metabolic tumor volume measured by $18 \mathrm{~F}-\mathrm{FDG} \mathrm{PET} / \mathrm{CT}$ can predict the outcome of osteosarcoma of the extremities. J Nucl Med 54: 1725-1732.

18. Lee JW, Kang CM, Choi HJ, Lee WJ, Song SY, et al. (2014) Prognostic value of metabolic tumor volume and total lesion glycolysis on preoperative 18F-FDG $\mathrm{PET} / \mathrm{CT}$ in patients with pancreatic cancer. J Nucl Med 55: 898-904.

19. Pak K, Cheon GJ, Nam HY, Kim SJ, Kang KW, et al. (2014) Prognostic value of metabolic tumor volume and total lesion glycolysis in head and neck cancer: A systematic review and meta-analysis. J Nucl Med 55: 884-890.

20. Isiguzo M, Brunken R, Tchou P, Xu M, Culver DA (2011) Metabolism-perfusion imaging to predict disease activity in cardiac sarcoidosis. Sarcoidosis Vasc Diffuse Lung Dis 28: 50-55.

21. Ahmadian A, Brogan A, Berman J, Sverdlov AL, Mercier G, et al. (2014) Quantitative interpretation of FDG PET/CT with myocardial perfusion imaging increases diagnostic information in the evaluation of cardiac sarcoidosis. J Nuc Cardiol 21: 925-939. 
Citation: Maruoka Y, Nagao M, Baba S, Isoda T, Kitamura Y, et al. (2017) Evaluation of Response to Steroid Therapy for Cardiac Sarcoidosis Using Volumetric Analysis of ${ }^{18}$ F-FDG PET/CT. J Bioanal Biomed 9: 229-234. doi:10.4172/1948-593X.1000184

22. Betensky BP, Tschabrunn CM, Zado ES, Goldberg LR, Marchlinski FE, et al. (2012) Long-term follow-up of patients with cardiac sarcoidosis and implantable cardioverter-defibrillators. Heart Rhythm 9: 884-891.

23. Mc Ardle BA, Birnie DH, Klein R, de Kemp RA, Leung E, et al. (2013) Is there an association between clinical presentation and the location and extent of myocardial involvement of cardiac sarcoidosis as assessed by $18 \mathrm{~F}$-fluorodoexyglucose positron emission tomography? Circ Cardiovasc Imaging 6: 617-626.

24. Morooka M, Moroi M, Uno K, Ito K, Wu J, et al. (2014) Long fasting is effective in inhibiting physiological myocardial 18F-FDG uptake and for evaluating active lesions of cardiac sarcoidosis. EJNMMI Res 4: 1. 